\title{
The Sc-GAP makeover flap: eliminating the need for position changes in gluteal flap breast reconstruction
}

\author{
Ennie Bijkerk ${ }^{1,2}$ - René R. W. J. van der Hulst ${ }^{1,2} \cdot$ Arno Lataster $^{3}$ - Stefania M. H. Tuinder ${ }^{1,2}$
}

Received: 13 January 2020 / Accepted: 17 March 2020 / Published online: 3 April 2020

(C) The Author(s) 2020

\begin{abstract}
Background The gluteal region is one of the many alternative donor sites for autologous breast reconstruction. However, the harvest of the gluteal flap is rather difficult, and the major drawback of gluteal flaps has been the need for position changes for flap harvest and inset. A new approach of a gluteal flap is introduced, based on the septocutaneous perforators of the superior gluteal artery: the septocutaneous gluteal artery perforator (Sc-GAP) makeover flap.

Methods A prospective study was performed in Maastricht University Medical Center between January 2018 and December 2019. Patients who underwent a Sc-GAP makeover flap breast reconstruction in the Maastricht University Medical Center and have had preoperative magnetic resonance angiography (MRA) of the abdomen between January 2018 and June 2019 were included.

Results Nine patients underwent breast reconstruction with thirteen Sc-GAP makeover flaps, of which nine flaps were innervated. Indications were the abdomen not being available as a donor site $(n=4)$ or the flank region was preferred as a donor site by the patient $(n=5)$. The total operative time was $430 \mathrm{~min}$ on average (range 311-683). Mean flap weight was $638 \mathrm{~g}$ (range 370-1004) and the mean ischemia time was $53 \pm 9.96 \mathrm{~min}$. Coupler size used was $2.0-2.5 \mathrm{~mm}$. All flaps survived.

Conclusion The Sc-GAP makeover flap overcomes the disadvantages of the conventional gluteal flaps, especially by eliminating the need for position changes during the reconstruction procedure. It is a reliable flap that provides sufficient volume and good esthetic outcomes.

Level of evidence: Level IV, therapeutic study.
\end{abstract}

Keywords Breast cancer $\cdot$ Autologous breast reconstruction $\cdot$ Gluteal flap $\cdot$ Septocutaneous perforator $\cdot$ Superior gluteal artery

\section{Introduction}

The deep inferior epigastric perforator (DIEP) flap is worldwide accepted as the first choice donor site for autologous breast reconstruction [1]. However, autologous breast reconstruction in patients with insufficient abdominal fat or those who have previously undergone a DIEP flap breast reconstruction or abdominoplasty is often challenging. Breast reconstruction requires an individualized approach and

Stefania M. H. Tuinder

s.tuinder@mumc.nl

1 Department of Plastic and Reconstructive Surgery, Maastricht University Medical Center, Maastricht, Netherlands

2 GROW - School for Oncology and Developmental Biology, Maastricht University Medical Center, Maastricht, Netherlands

3 Department of Anatomy and Embryology, Maastricht University, Maastricht, Netherlands depending on the patients' wishes and body habitus, a broad range of alternative donor sites can be considered.

In the literature, the gluteal region and flank have been well described as a donor site for autologous breast reconstruction, providing a variety of flaps based on different perforators [2-5]. In 1975, the superior gluteal myocutaneous free flap was introduced by Fujino et al. [6], which was later refined to the superior gluteal artery perforator (S-GAP) flap without muscle sacrifice by Allen and Tucker [7]. In 2008, Tansatit et al. [8] performed an anatomical study and stated that the penetrating branch, coming from the superficial branch of the superior gluteal artery (SGA), is recommended to use for the harvest of an S-GAP free flap. After Tuinder et al. first reported the existence of septocutaneous perforators between the gluteus maximus and medius muscles in an anatomical study in 2008 [9], the first clinical experiences with a flap based on these septocutaneous perforators for microsurgical breast reconstruction were reported several years after by our group as well as by Rad et al. [10,11]. These septocutaneous 
perforators run in the septal plane between the gluteus maximus and medius muscles, and their course obviates the need for intramuscular dissection. Moreover, they are consistently present [9]. However, one of the major drawbacks of the gluteal flaps has always been the need for position changes for flap harvest and inset.

The purpose of this study is to introduce a new gluteal free flap for breast reconstruction, based on the septocutaneous perforators of the superficial branch of the SGA as the pedicle, but more ventrally located than the conventional S-GAP flap. This location offers the advantage of positioning the patient in supine position for dissection of the perforating septocutaneous branch of the SGA.

\section{Materials and methods}

\section{Patients}

A prospective study was performed in Maastricht University Medical Center between January 2018 and December 2019. Patients who underwent a Sc-GAP makeover flap breast reconstruction in the Maastricht University Medical Center and have had preoperative magnetic resonance angiography (MRA) of the abdomen between January 2018 and June 2019 were included. Informed consent was obtained.

\section{Anatomy and preoperative landmarks}

The gluteal region consists of one deep and one superficial muscle layer. The deep layer is composed of the gluteus medius, minimus, piriformis, triceps coxae and quadratus femoris muscles, and the superficial layer of the gluteus maximus muscle. The fascia that covers the cranial part of the gluteus medius muscle is thick and dens. The caudal part of the gluteus medius muscle is covered by the gluteus maximus muscle. The gluteus maximus and medius muscles are separated by a thin areolar fascia. The gluteus maximus muscle is thick and prominent on the lateral part, but is quite thin in the craniomedial part. This difference in thickness facilitates finding the septal plane at a distance of approximately $7 \mathrm{~cm}$ from the midline.

Both the superior and inferior gluteal arteries (SGA and IGA) originate from the internal iliac artery at the level of the greater sciatic foramen. The SGA originates from the posterior trunk and is the largest branch of the internal iliac artery. It leaves the pelvis via the suprapiriform foramen, and at that point, it branches into a deep branch and a superficial branch. The superficial branch has a septocutaneous course, in the septal plane between the gluteus maximus and medius muscles, and provides three clinically important perforators: muscular perforators that supply the gluteus maximus muscle, and musculocutaneous and septocutaneous perforators that supply the subcutaneous tissue and skin. The musculocutaneous perforators of the superficial branch of the SGA are most commonly used as the pedicle in conventional S-GAP flaps for breast reconstruction [8]. The septocutaneous perforators arise from the fascia of the gluteus medius at the superolateral edge of the gluteus maximus and supply the subcutaneous tissue and skin. The musculocutaneous perforators from the deep branch of the SGA run through the gluteus maximus and medius muscles and are therefore not suitable as a Sc-GAP perforator because of a complex intramuscular dissection. At the point where the deep and superficial branches of the SGA meet, a caput medusae is located, which is a network of large, fragile veins. Dissection of the pedicle should stop before the caput medusa.

\section{Preoperative planning}

Preoperatively all patients underwent physical examination and MRA of the abdomen. Physical examination shows that the abdominal region is not suitable as a donor site, and the patient has enough subcutaneous tissue around the flanks. The MRA examinations are assessed to evaluate if the patient has suitable septocutaneous perforators originating from the SGA. The MRA examinations are performed on a 1.5 Tesla MRI scanner (Ingenia, Philips Healthcare, Best, the Netherlands), using a four-channel body coil and gadobutrol $1.0 \mathrm{mmol} / \mathrm{L}$ (Gadovist, Bayer Healthcare Pharmaceuticals, Berlin, Germany) as a contrast agent.

Preoperative drawing of the flap is performed 1 day prior to surgery, when the patient is admitted in the hospital. It is important that the patient is in supine position, as she will be during surgery. A hand-held Doppler device is used to locate the perforators that were selected as suitable perforators on MRA. The anterior border of the ASIS is the anterior border of the flap. The ellipse-shaped flap is horizontally drawn around the perforator to posterior. The anatomical landmarks and septocutaneous perforators can be confirmed using color Doppler (Esaote MyLab 25 Color Doppler and LA 532, 4 $13 \mathrm{MHz}$ probe). The pinch test is used to assess the maximal width of the flap. The flap is located higher and more ventrally than the original Sc-GAP flap.

\section{Surgical technique}

All procedures were performed by one surgeon (ST). The patients were positioned in supine position with sterile dressings. The arm is tucked with a draw sheet, which is secured under the patient, not the mattress [12]. It hangs slightly below the thigh for good access to the flap. The arm can also be positioned in 90 degrees abduction. A cushion was placed behind the back at the lumbar lordosis level, which provided better access for dissection of the vascular pedicle of the flap (Fig. 1). Two teams worked simultaneously together during 
surgery. One team worked on the dissection of the Sc-GAP makeover flap, and the other team performed mastectomy or removed implants if necessary and prepared the recipient vessels for microsurgical anastomosis.

The dissection of the flap started ventrally at the level of the anterior border of the anterior superior iliac spine (ASIS) until the fascia of the gluteal medius muscle is reached (Fig. 2a). The flap is lifted from anterior to posterior until the septum between the gluteus maximus and medius muscles is reached (Fig. 2b). The fascia is opened to reach the septal plane (Fig. 2c). The septocutaneous perforators are dissected and followed back until the caput medusae is reached, where the perforator is clipped and dissected. If it is possible, more than one perforator is dissected. Otherwise, the most lateral perforator is selected. This will determine the length of the pedicle: the higher the perforator, the shorter the pedicle. The length of the pedicle should be at least 6 to $9 \mathrm{~cm}$ (Fig. 2d).

For a more comprehensive description of the surgical technique, tips and tricks and a detailed description of the gluteal anatomy, we kindly refer you to the book chapter "Septocutaneous Gluteal Artery Perforator Flap in Breast Reconstruction" (p. 219-230) in "Breast Reconstruction Modern and Promising Surgical Techniques" by Springer Nature (https://link.springer.com/chapter/10.1007/978-3-03034603-4_23\#citeas).

A microscopical end-to-end anastomosis is performed. The arterial anastomosis is handmade, and the venous anastomosis is created using a coupler device. After flap shaping and inset, the perforating Doppler signals are confirmed and marked for monitoring the flap. The donor site is cranially undermined, and the wound edges are approximated using quilting sutures. The patients were followed up for esthetic outcomes and complications.



Fig. 1 Patient in supine position with a cushion under the hip at the level of the lumbar lordosis
Continuous variables are presented as means and ranges or median and ranges. Categorical variables are presented as total numbers.

\section{Results}

\section{Study population}

From January of 2018 to December of 2019, thirteen Sc-GAP makeover flaps were performed for gluteal breast reconstruction in 9 patients, with a mean age of 47 years. Patient characteristics are shown in Table 1. None of the patient actively smoked prior to the surgery.

The indications for choosing the Sc-GAP makeover flap are summarized in Table 1. Most patients preferred the gluteal region as the donor site instead of the abdomen $(n=5)$. In the remaining patients $(n=4)$, the abdomen was no longer available due to previous abdominoplasties $(n=1)$ or DIEP flap breast reconstructions $(n=3)$. Of each different indication, one case is discussed.

\section{Operative details}

The total operative time for unilateral reconstructions was 334 min on average (range 311-390), and for bilateral cases, it was $550 \mathrm{~min}$ (range 474-683). Mean flap weight was $638 \mathrm{~g}$ (range 370-1004) and the mean ischemia time was $53 \pm$ $9.96 \mathrm{~min}$. Coupler size used was $2.0-2.5 \mathrm{~mm}$. In 9 flaps, sensory nerve coaptation was performed.

\section{Clinical cases}

\section{Patient 1 - Patient's preference}

A 40-year-old woman with a BMI of 24.44 came to the outpatient clinic in March 2018 after she underwent a bilateral mastectomy followed by implant-based breast reconstruction (IBBR) 4 years before (Fig. 3a). The patient had extensive pain due to her silicone breast implants. During preoperative counseling, patient stated that she absolutely did not want a scar in her abdominal region. Her own suggestion was to use the flanks as a donor site. In September 2018, she underwent bilateral tertiary autologous breast reconstruction with ScGAP makeover flaps from both flanks. Three days postoperatively, she was taken back to the operating room because of venous congestion of the Sc-GAP makeover flap on the right side due to a hematoma. Six days later, she was released from the hospital, after a total hospital stay of 9 days. Both flaps survived, but secondary corrections were necessary to achieve symmetry (Fig. 3b). 
Fig. 2 Step-by-step surgical technique. a Starting dissection at the ASIS until the fascia of the gluteus medius muscle is reached. b Raise the flap from ventral to dorsal until the septum between the gluteus maximus and medius muscles is reached. c Open the septum and dissect the perforators back until the caput medusae. d The length of the pedicle shown here is approximately $8-9 \mathrm{~cm}$ long
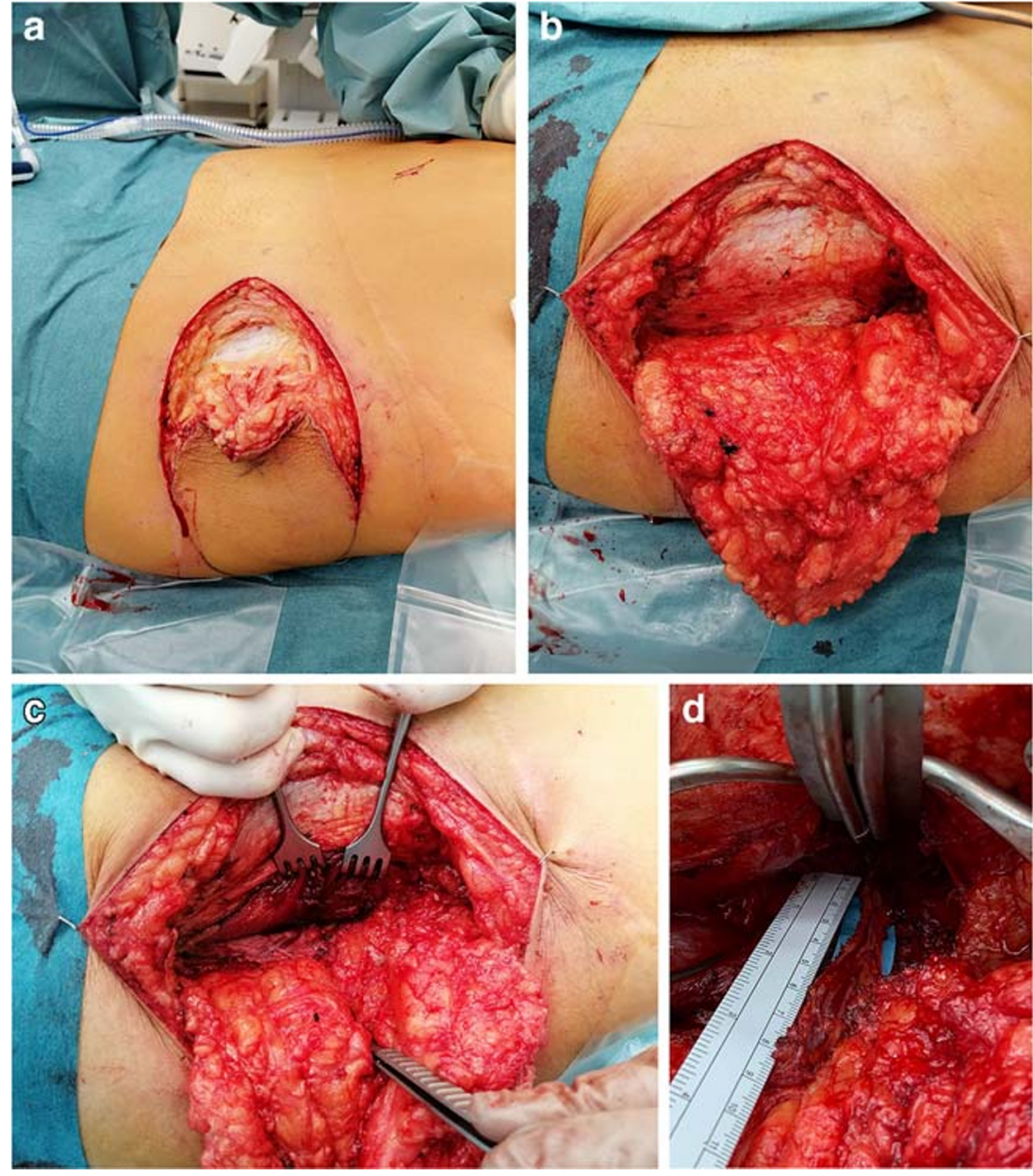

\section{Patient 2-Abdominoplasty}

Table 1 Patient demographics

\begin{tabular}{ll} 
Total no. of patients & 9 \\
Total no. of Sc-GAP makeover flaps & 13 \\
Age in years (mean, range) & $47(36-63)$ \\
BMI in $\mathrm{kg} / \mathrm{m}^{2}$ (mean, range) & $26.54(21.74-33.46)$ \\
Type of reconstruction (patients) & \\
$\quad$ Unilateral & 5 \\
$\quad$ Bilateral & 4 \\
Timing of reconstruction (flaps) & \\
Primary & 4 \\
Secondary & 7 \\
$\quad$ Tertiary & 2 \\
Indication for Sc-GAP makeover flap (patients) & \\
$\quad$ First choice (abdomen) not available & 4 \\
$\quad$ Previous abdominoplasty & 1 \\
$\quad$ Previous DIEP flap breast reconstruction & 3 \\
Patient preference & 5 \\
Hospital stay in days (mean, range) & $6(4-9)$ \\
Follow-up in days (median, IQR) & $158(52-394)$ \\
\hline
\end{tabular}

A 63-year-old woman who previously underwent an abdominoplasty was referred to the outpatient clinic of General Surgery after an abnormality was found in her right breast during the national breast cancer screening program. The patient initially underwent a lumpectomy of the right breast, which unfortunately was not radical and was therefore planned for amputation with immediate reconstruction with the Sc-GAP makeover flap. Preoperatively the flap was drawn bilaterally for a stacked reconstruction, because initially it was thought that a unilateral flap would not provide enough volume to achieve symmetry with the contralateral breast (Fig. 4a). Intraoperatively one flap showed to be sufficient for volume symmetry. During surgery, the vascularization of the flap was verified using indocyanine green (ICG).

Three days postoperatively, she developed a pulmonary embolism, which was treated with apixaban for 3 months. Due to the anticoagulants, she experienced some persistent bleeding from the surgical wounds. However, it was not excessive and after 2 months, all wounds were healed. She was pleased with the results (Fig. 4b). 
Fig. 3 3a Preoperative photos after previous bilateral mastectomy and implant-based breast reconstruction. $3 \mathrm{~b}$ Postoperative photos of the reconstructed breasts, 6 months after surgery
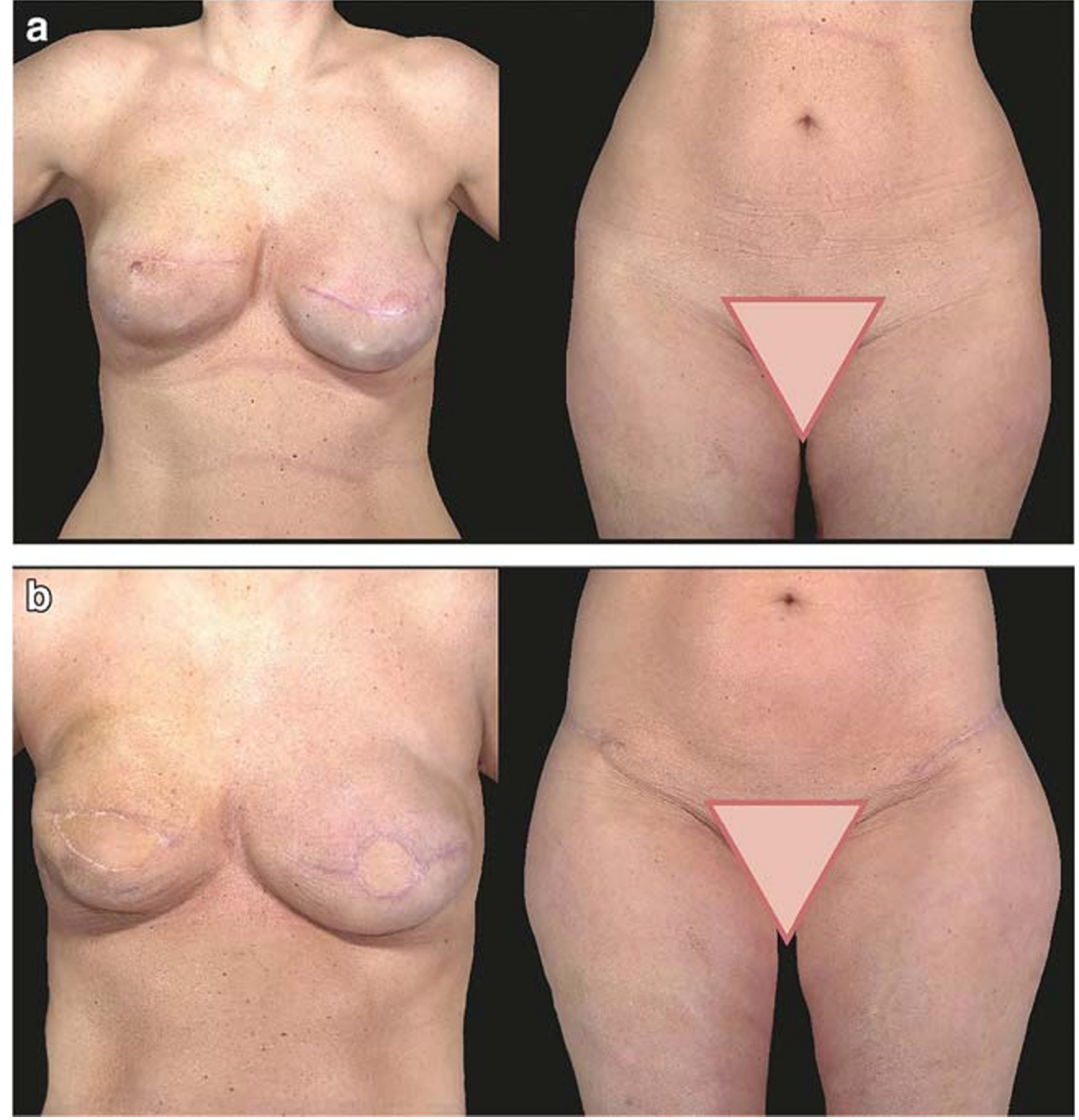

\section{Patient 3-DIEP flap breast reconstruction}

A 52-year-old woman developed breast cancer on the right breast in February 2019. She underwent a DIEP flap breast reconstruction of the left breast in 2016 after developing breast cancer. Therefore, the abdomen was no longer suitable as a donor site. The lateral thigh and flank were considered alternative donor sites (Fig. 5a). The patient preferred the flank and preoperative imaging showed adequate septocutaneous gluteal artery perforators with good caliber.

She underwent a mastectomy and sentinel node followed by immediate Sc-GAP makeover flap breast reconstruction of the right breast. Her postoperative course was uneventful. A secondary correction surgery was planned to achieve
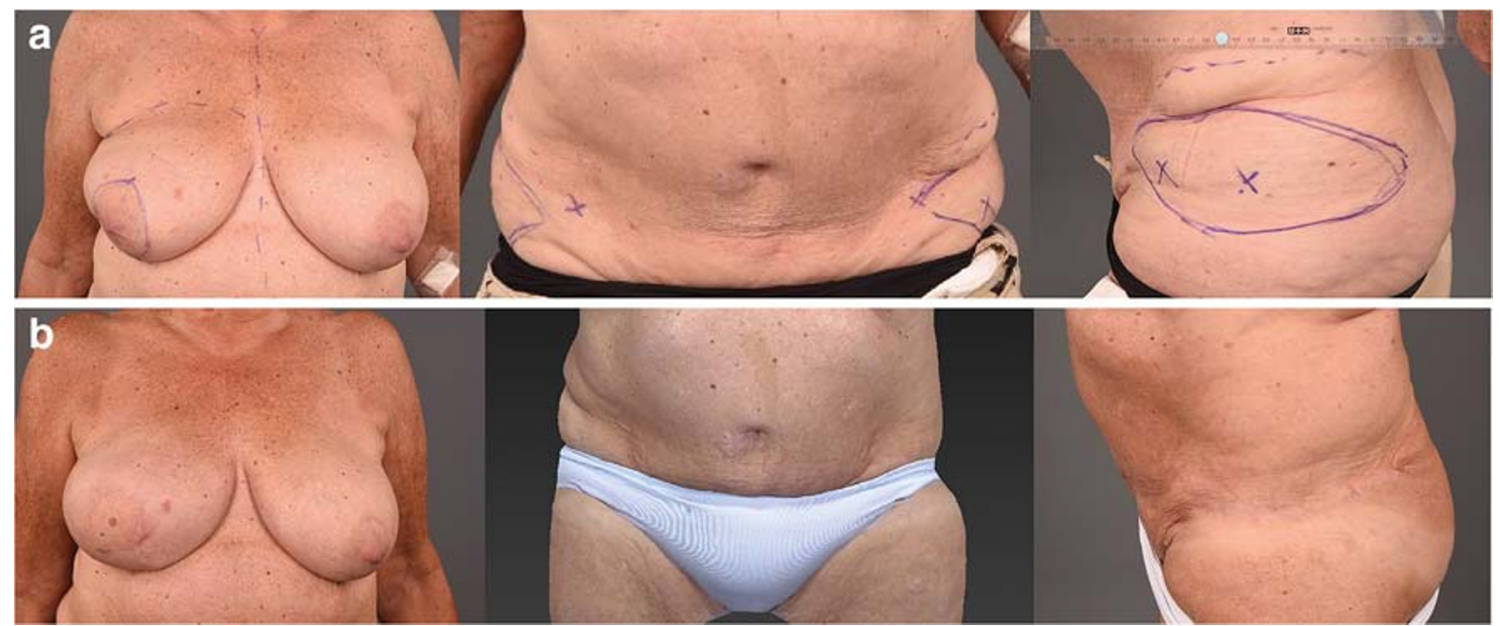

Fig. 4 a Preoperative photos after a previously abdominoplasty and lumpectomy with bilateral flap drawing. b Postoperative photos of the reconstructed breast on the right side and the donor site (left flank) 9 months after surgery 
Fig. 5 a Preoperative photo of the patient, before she underwent the DIEP flap breast reconstruction of the left breast. b Postoperative photos after a DIEP flap breast reconstruction of the left breast and a Sc-GAP makeover flap breast reconstruction coming from the right flank on the righthand side, including a lateral view of the donor site
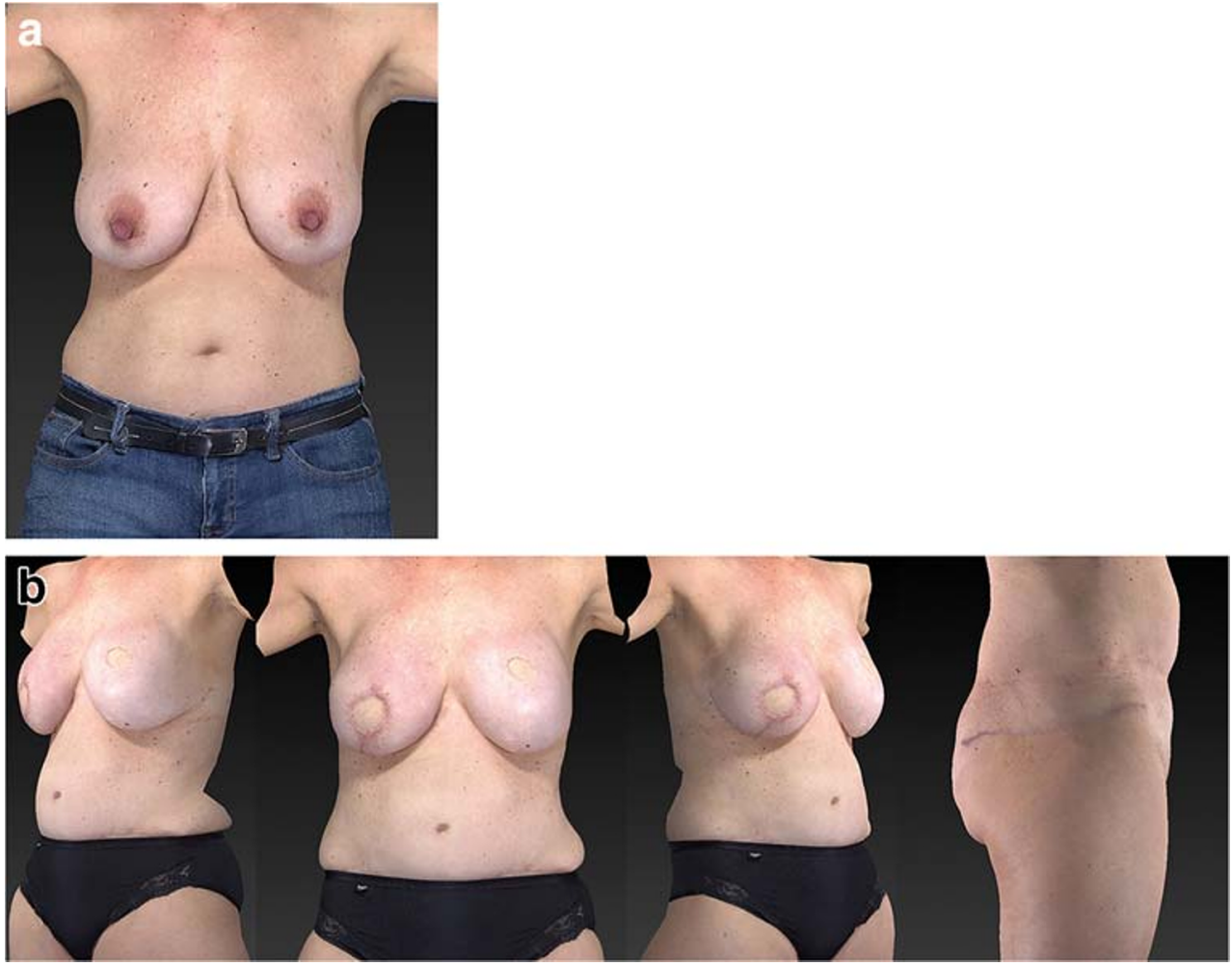

symmetry of the breasts by removing the skin islands. However, even without secondary corrections, a similar shape, volume, projection, and ptosis can be seen between the DIEP flap and the Sc-GAP makeover flap (Fig. 5b).

\section{Complications}

One flap required re-exploration, 3 days postoperatively, because of venous congestion due to a hematoma in the breast, which resulted in a viable flap. One patient developed a persistent seroma at the donor site, lasting 85 days. One patient had wound problems of the mastectomy skin that did not require any surgical intervention. One patient developed a pulmonary embolism after surgery, for which she was treated with anticoagulants. No further re-interventions were necessary, and all flaps survived.

\section{Projection of subcutaneous tissue}

A comparison between the projection of subcutaneous tissue of the flaps that are commonly used for autologous breast reconstruction and can be harvested with the patient in supine position is presented in Table 2 and Fig. 6. The preoperative MRA examinations of the study population were used. The mean projection of the Sc-GAP makeover flap was $61.6 \mathrm{~mm}$ and therefore provided the most subcutaneous tissue of the investigated donor sites. The DIEP flap had only $24.8 \mathrm{~mm}$ thickness of the subcutaneous tissue. Of course it needs to be taken in consideration that 4 of the 9 patients previously underwent an abdominoplasty or a DIEP flap. However, two of these patients had thicker abdominal tissue after a DIEP flap breast reconstruction (varying from 26.5 to $30.6 \mathrm{~mm}$ ) compared with the patients who did not undergo abdominal surgery before (mean $25.5 \mathrm{~mm}$ ).

The Sc-GAP makeover flap provides adequate projection of subcutaneous tissue to reconstruct a breast, even without folding the flap. Of course the projection of the subcutaneous tissue alone does not determine the total volume of the flap, but it provides the surgeon an indication what kind of tissue is available in this region.

Table 2 Subcutaneous projection per donor site

\begin{tabular}{ll}
\hline \multicolumn{1}{c}{ Flap } & Projection in mm \\
\hline DIEP & $24.8 \pm 5.6$ \\
Previous DIEP or abdominoplasty $(n=4)$ & $20.4 \pm 5.3$ \\
No previous abdominal surgeries $(n=5)$ & $25.5 \pm 6.1$ \\
LTP & $59.5 \pm 11.6$ \\
PAP & $31.7 \pm 6.1$ \\
TUG & $36.8 \pm 6.0$ \\
Sc-GAP makeover & $61.6 \pm 15.7$ \\
\hline
\end{tabular}

All data is shown as mean and standard deviation. The projection measurements are shown in millimeters 
Fig. 6 The differences in projection of the subcutaneous fat of commonly used flaps that can be harvested with the patient in supine position

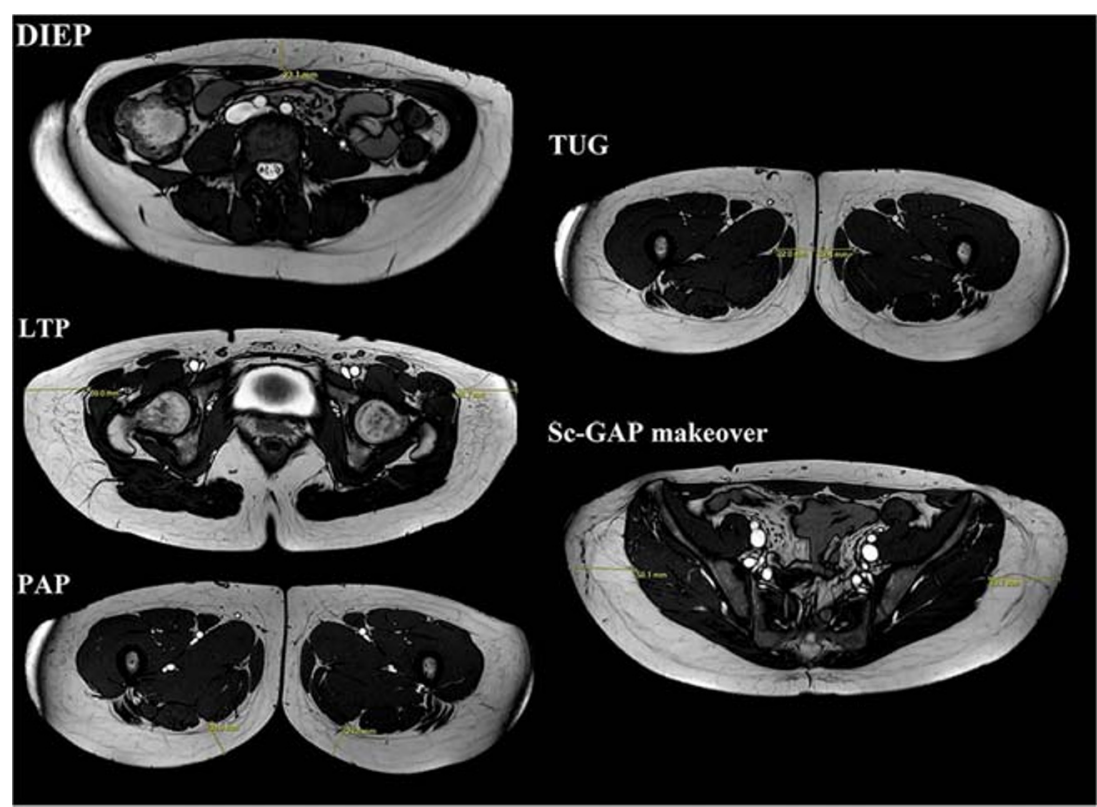

\section{Discussion}

The aim of the current study was to analyze our first experiences with the Sc-GAP makeover flap and introduce it as a simplified approach in gluteal flaps for autologous breast reconstruction. The gluteal region is one of the alternative donor sites for those patients who are not eligible for a DIEP flap. However, the harvest of the S-GAP flap is rather difficult and requires advanced microsurgical expertise [5]. Therefore, Tuinder et al. introduced the Sc-GAP flap, which obviates the need for intramuscular dissection of the pedicle $[9,10]$. Despite the septocutaneous course of the perforators, the dissection of the pedicle remains challenging, and the gluteus maximus muscle needs to be elevated during the dissection for adequate visibility. Moreover, deformity of the gluteal region often results in a depression or dent in the buttock. However, the major drawback of the gluteal flap is that this technique requires position changes for flap harvest and flap

Table 3 Disadvantages of the gluteal flap for microsurgical breast reconstruction
Position changes required for flap harvest and inset

Simultaneous work not possible

Difficult dissection because of necessity of pulling up the gluteus maximus muscle and inferior approach of the pedicle

Scar position and shape

Contour deformity of the gluteal projection

S-shaped scar is difficult to hide underneath clothes
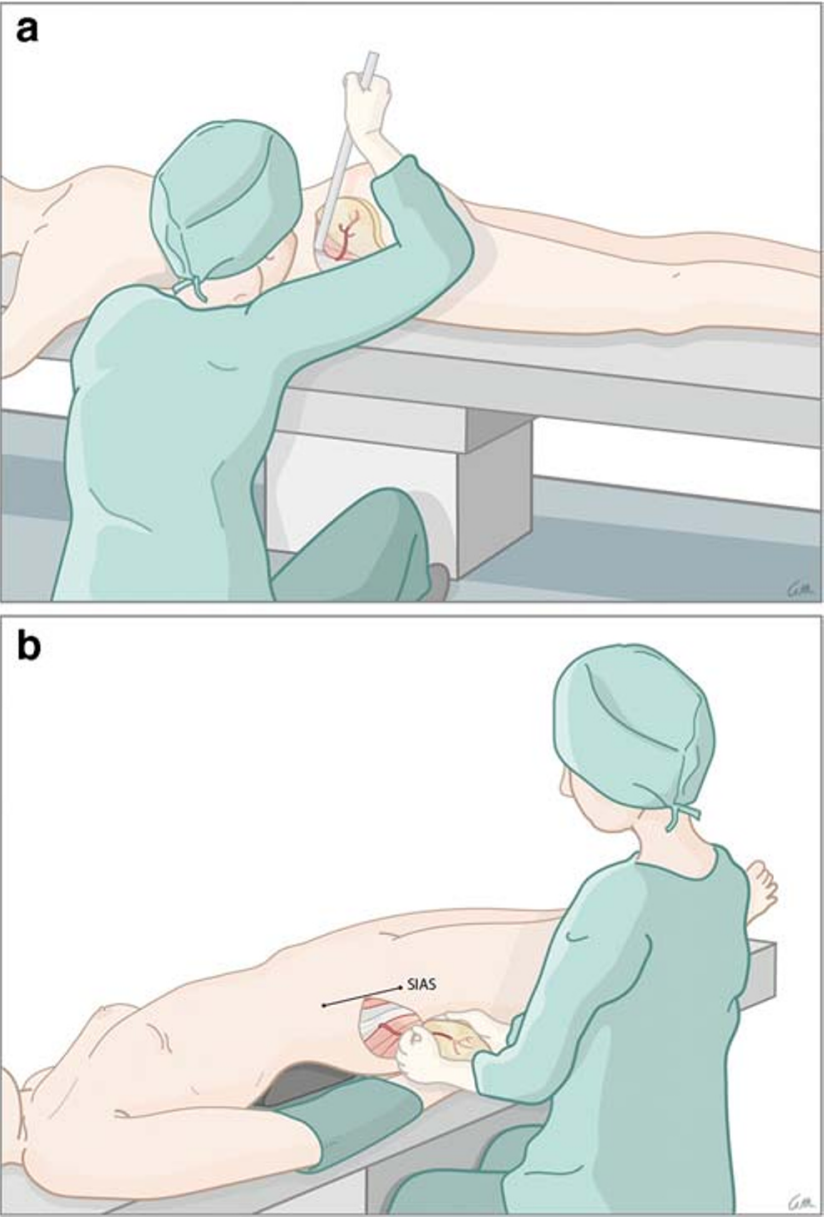

Fig. 7 A schematic overview of the positioning of the patient and the posture of the plastic surgeon during harvest of the conventional Sc-GAP flap a and the Sc-GAP makeover flap b 
inset. Consequently, the possibility for simultaneous work on the donor site and the thorax is averted.

The reasons why the gluteal flaps have been abandoned as a second choice in autologous breast reconstruction are summarized in Table 3. The Sc-GAP makeover flap offers the solution to overcome these disadvantages. First, the location of the flap is more ventrally. This enables flap harvest with the patient in supine position and, therefore, eliminates the need for position changes, which was previously considered to be unavoidable [11]. The proposed technique gives the advantage of a two- or three-teamed approach. The dissection of the pedicle is also facilitated. The conventional dissection of the Sc-GAP flap started cranially and continued caudally. Therefore, it was necessary to pull up the gluteus maximus muscle for adequate visibility, and often the gluteus maximus muscle was medially detached from the sacrum for optimal accessibility (Fig. 7a). In contrary to the dissection of the conventional Sc-GAP flap, the dissection of the Sc-GAP makeover flap starts ventrally, and continues to dorsally. Because the patient is in supine position, the flap falls down due to gravity, which enables adequate visibility into the septal plane between the gluteus maximus and medius muscles, without pulling on any muscles (Fig. 7b).

In addition, the location of the flap minimizes contour changes at the gluteal region, particularly regarding projection of the buttock. In contrary to the S-shaped scar of the Sc-GAP flap, the Sc-GAP makeover flap offers a straight scar, which makes it easier to hide underneath clothes (Fig. 8).
Another difference is that subcutaneous tissue of the ScGAP makeover flap is less stiff and firm than that of a conventional gluteal flap and more comparable to the subcutaneous tissue of the LTP flap. However, the Sc-GAP makeover flap also offers the possibility for cranial and caudal beveling of the donor site, which enhances the esthetic outcomes of the donor site without contour deformity of the buttock. This offers an advantage compared with the lateral thigh perforator (LTP) flap, where beveling the donor sites results in a significant contour deformity of the lateral thigh [13]. Not beveling the LTP flap results in rather "sharp" edges of the flap, giving it a more squared shape. Patients who underwent a LTP flap for breast reconstruction almost always desire secondary corrections with lipofilling of their breasts because of this squared shape. Beveling the Sc-GAP makeover flap results in smooth transition of the flap edges, like those of the DIEP flap, and ensures volume of the upper pole of the reconstructed breast [14]. Figure $5 \mathrm{~b}$ shows that the Sc-GAP makeover flap is actually similar to the DIEP flap, which is the first choice and can be considered the gold standard for autologous breast reconstruction. The shape, volume, projection, and ptosis of both breasts are comparable, emphasizing the strengths of the Sc-GAP makeover flap as an alternative, or even a first choice for autologous breast reconstruction.

The nomenclature of this flap has not yet been established. Rad et al. named the flap after the superolateral perforator that exits the septal plane: the lateral septocutaneous superior gluteal artery perforator (LSGAP), but reported a rate of 33\% of
Fig. 8 The differences in scar shape and scar location between the Sc-GAP flap a and the ScGAP makeover flap b
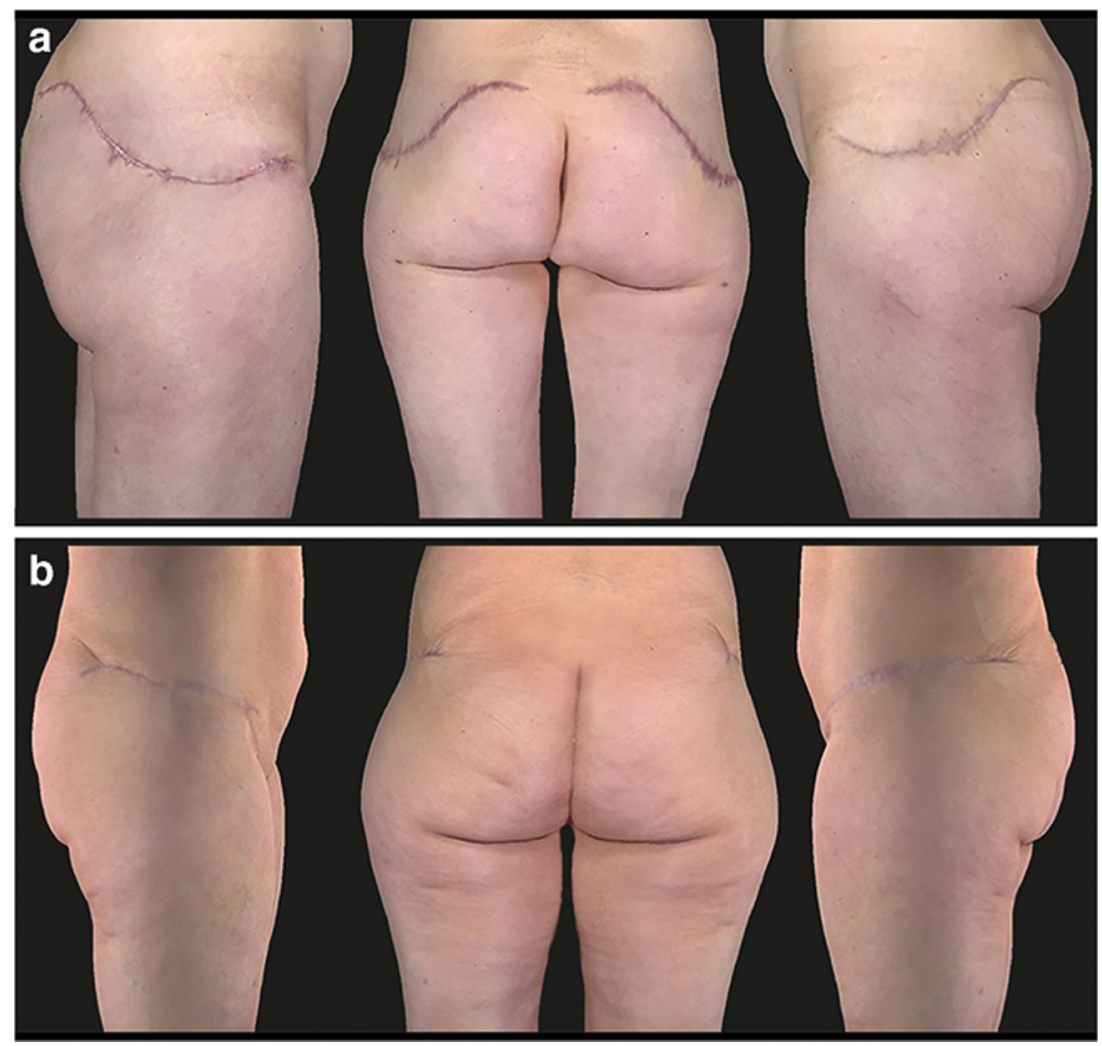
inadequate or absent septocutaneous perforators in their cadaveric dissections. Fade et al. uses the same abbreviation for a similar nomenclature: lateral superior gluteal artery perforator, as they were unable to confirm the consistent septocutaneous course of the consistently present superolateral perforator of the SGA $[11,15]$. Unlike the studies by Rad et al. and Fade et al., our anatomical study in 2008 demonstrated the consistent presence of septocutaneous perforators between the gluteus maximus and medius muscles [9]. As suggested by Kim et al. in 2004 [16] and established by Taylor et al. in 2012 [17], we prefer the perforator flap nomenclature based on anatomical principles, such as the true course of a perforator: the septocutaneous gluteal artery perforator (Sc-GAP) flap.

So far, our first experiences with the Sc-GAP makeover have shown that this flap is a viable and reliable flap to use for autologous breast reconstruction in those patients who are not eligible for a DIEP flap or have other indications for alternative donor sites. Only 1 out of 14 flaps (7.6\%) required re-exploration because of flap congestion due to hematoma, which is twice as high as the numbers for venous congestion in DIEP flaps at our institution, varying from $3.1-4.8 \%[18,19]$. The difference might be explained by the complexity of the DIEP flaps, as our institution is a referral center for autologous breast reconstruction, as well as the limited cases of Sc-GAP makeover flaps compared with DIEP flaps. No other major complications of the reconstructed breasts or donor sites were seen, and all flaps survived. Moreover, patients were satisfied with the result, even after the first stage without any correction surgeries.

\section{Conclusion}

The Sc-GAP makeover flap is a reliable flap for autologous breast reconstruction and provides sufficient volume with good esthetic outcomes and projection of the breasts. The Sc-GAP makeover flap brings back the gluteal flap as a second choice for autologous breast reconstruction, as it avoids all the reasons why these flaps have been abandoned. The simplified approach enables more reconstructive but experienced surgeons to perform the technique and offers it as an alternative for those women in need for an individualized reconstructive plan.

Acknowledgments We would like to acknowledge the contribution of Greet Mommen of the department of Anatomy of the Maastricht University for the figures in this publication (http://www.greetmommen.be).

\section{Compliance with ethical standards}

Conflict of interest Ennie Bijkerk, René R. W. J. van der Hulst, Arno Lataster and Stefania M. H. Tuinder declare that they have no conflict of interest.
Ethical approval The study was approved by the institutional Medical Ethical Committee of Maastricht University and the Maastricht University Medical Center and performed in accordance with the ethical standards of the Declaration of Helsinki.

Informed consent Written informed consent was obtained from the patients.

Open Access This article is licensed under a Creative Commons Attribution 4.0 International License, which permits use, sharing, adaptation, distribution and reproduction in any medium or format, as long as you give appropriate credit to the original author(s) and the source, provide a link to the Creative Commons licence, and indicate if changes were made. The images or other third party material in this article are included in the article's Creative Commons licence, unless indicated otherwise in a credit line to the material. If material is not included in the article's Creative Commons licence and your intended use is not permitted by statutory regulation or exceeds the permitted use, you will need to obtain permission directly from the copyright holder. To view a copy of this licence, visit http://creativecommons.org/licenses/by/4.0/.

\section{References}

1. Healy C, Allen RJ Sr (2014) The evolution of perforator flap breast reconstruction: twenty years after the first DIEP flap. J Reconstr Microsurg 30(2):121-125

2. Colebunders B, Depypere B, Van Landuyt K (2016) The dog-ear flap as an alternative for breast reconstruction in patients who have already undergone a DIEAP flap. J Plast Reconstr Aesthet Surg 69(5):594-597

3. Elliott LF, Hartrampf CR Jr (1998) The Rubens flap. The deep circumflex iliac artery flap. Clin Plast Surg 25(2):283-291

4. Hamdi M, Craggs B, Brussaard C, Seidenstueker K, Hendrickx B, Zeltzer A (2016) Lumbar artery perforator flap: an anatomical study using multidetector computed tomographic scan and surgical pearls for breast reconstruction. Plast Reconstr Surg 138(2):343-352

5. Hunter C et al (2016) Superior gluteal artery perforator flap: the beauty of the buttock. Ann Plast Surg 76(Suppl 3):S191S195

6. Fujino T, Harasina T, Aoyagi F (1975) Reconstruction for aplasia of the breast and pectoral region by microvascular transfer of a free flap from the buttock. Plast Reconstr Surg 56(2):178-181

7. Allen RJ, Tucker C Jr (1995) Superior gluteal artery perforator free flap for breast reconstruction. Plast Reconstr Surg 95(7):1207-1212

8. Tansatit T, Chokrungyaranont P, Sanguansit P, Wanidchaphloi S (2008) Anatomical study of the superior gluteal artery perforator (S-GAP) for free flap harvesting. J Med Assoc Thail 91(8):1244 1249

9. Tuinder $\mathrm{S}$ et al (2008) Superior gluteal artery perforator flap based on septal perforators: preliminary study. Plast Reconstr Surg 122(5):146e-148e

10. Tuinder S, Chen CM, Massey MF, Allen RJ Sr, van der Huist R (2011) Introducing the septocutaneous gluteal artery perforator flap: a simplified approach to microsurgical breast reconstruction. Plast Reconstr Surg 127(2):489-495

11. Rad AN, Flores JI, Prucz RB, Stapleton SM, Rosson GD (2010) Clinical experience with the lateral septocutaneous superior gluteal artery perforator flap for autologous breast reconstruction. Microsurgery 30(5):339-347

12. Positioning the Patient for Surgery. Chapter 5, p 145. Jones \& Bartlett Learning, LLC, an Ascend Learning Company 
13. Tuinder SMH, Beugels J, Lataster A, de Haan MW, Piatkowski A, Saint-Cyr M, van der Hulst R, Allen RJ (2018) The lateral thigh perforator flap for autologous breast reconstruction: a prospective analysis of 138 flaps. Plast Reconstr Surg 141(2): 257-268

14. Sharma HR, Rozen WM, Mathur B, Ramakrishnan V (2019) 100 steps of a DIEP flap-a prospective comparative cohort series demonstrating the successful implementation of process mapping in microsurgery. Plast Reconstr Surg Glob Open 7(1):e2016

15. Fade G et al (2013) Anatomical basis of the lateral superior gluteal artery perforator (LSGAP) flap and role in bilateral breast reconstruction. J Plast Reconstr Aesthet Surg 66(6):756-762

16. Kim JT (2005) New nomenclature concept of perforator flap. Br J Plast Surg 58(4):431-440
17. Taylor GI, Rozen WM, Whitaker IS (2012) Establishing a perforator flap nomenclature based on anatomical principles. Plast Reconstr Surg 129(5):877e-879e

18. Beugels J, Bod L, van Kuijk S, Qiu SS, Tuinder SMH, Heuts EM, Piatkowski A, van der Hulst R (2018) Complications following immediate compared to delayed deep inferior epigastric artery perforator flap breast reconstructions. Breast Cancer Res Treat 169(2): 349-357

19. Beugels $\mathrm{J}$ et al (2016) Complications in unilateral versus bilateral deep inferior epigastric artery perforator flap breast reconstructions: a multicentre study. J Plast Reconstr Aesthet Surg 69(9):1291-1298

Publisher's note Springer Nature remains neutral with regard to jurisdictional claims in published maps and institutional affiliations. 